\title{
GENERATION AND APPLICATION OF ENERGETIC HEAVY ION AND X-RAY VIA 12TW50FS LASER-SOLID INTERACTION
}

\author{
K. Kinoshita, T. Ohkubo, H. Okuda, T. Kobayashi, K. Yoshii, T. Watanabe, T. Ueda, \\ M. Uesaka, NERL, University of Tokyo, Tokai, Naka, Ibaraki, 319-1188 Japan
}

\begin{abstract}
Energetic ions and ultrashort X-rays were generated by an intense ultrashort laser interacting with a copper target. Fast ions up to $3 \mathrm{MeV}$ were observed with CR-39 nuclear track detector sheets when 7 TW laser was used. $\mathrm{Cu}^{12+} \sim$ $\mathrm{Cu}^{17+}$ ions with the energy from $50 \mathrm{keV}$ to $220 \mathrm{keV}$ were detected with a Thomson parabola detector in the case of the laser power of $2.5 \mathrm{TW}$. Time-resolved X-ray diffraction was carried out using ultrashort X-rays generated from the copper target irradiated by the intense laser pulses. A change of diffraction patterns was observed in the X-ray diffraction from a GaAs(111) crystal irradiated by the ultrashort laser pulses.
\end{abstract}

\section{INTRODUCTION}

Recent rapid progress in ultraintense lasers has brought promising applications such as particle acceleration and Xray generation. Nowadays laser intensity of $10^{16}$ to $10^{18}$ $\mathrm{W} / \mathrm{cm}^{2}$ is achievable by focusing a high power laser. Such intense laser pulses can easily generate hot plasmas by interaction with solid targets [1]. Those plasmas can be sources of various kinds of radiation such as energetic ions and ultrashort X-rays. In this study, we generate heavy ion beams and X-ray pulses using 12TW50fs laser focused on a $\mathrm{Cu}$ target. The ion beam parameters such as the number and energy spectrum are measured. The ion beam may be used as a source of accelerators, or will be applied to observe the cascade radiation damage of nuclear materials. The generated X-rays are used for time-resolved X-ray diffraction experiments to investigate ultrafast atomic motion in crystals irradiated by ultrashort lasers. Temporal evolution of diffraction patterns is obtained in the picosecond time scale. The changes of diffraction profiles are compared with numerical calculations.

\section{ENERGETIC HEAVY ION GENERATION}

Figure 1 shows the experimental setup for ion generation. This experiment was carried out at NERL, with 12 TW Ti:Sapphire chirped pulse amplification laser system which produces the laser pulses having energies of up to $600 \mathrm{~mJ}$ at a wavelength of $790 \mathrm{~nm}$ and temporal duration of $50 \mathrm{fs}$ at $10 \mathrm{~Hz}$. The laser beam is p-polarized and is focused onto a $\mathrm{Cu}$ target with an $\mathrm{f}=1.5$ off-axis parabolic mirror at an incident angle $60^{\circ}$ from the target normal. The maximum laser intensity on the target is approximately 7

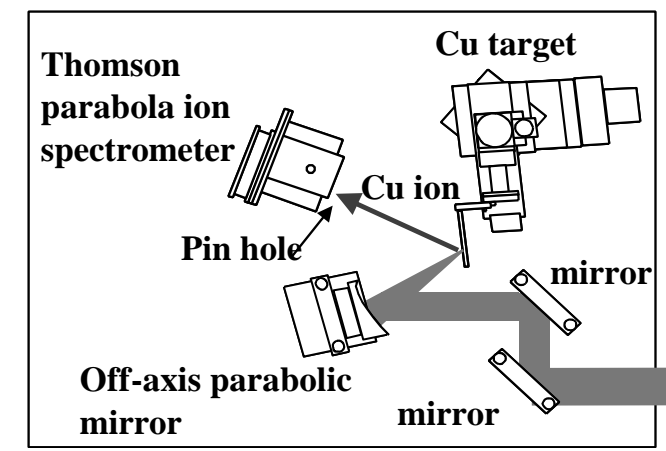

Figure 1: Experimental setup for ion generation.

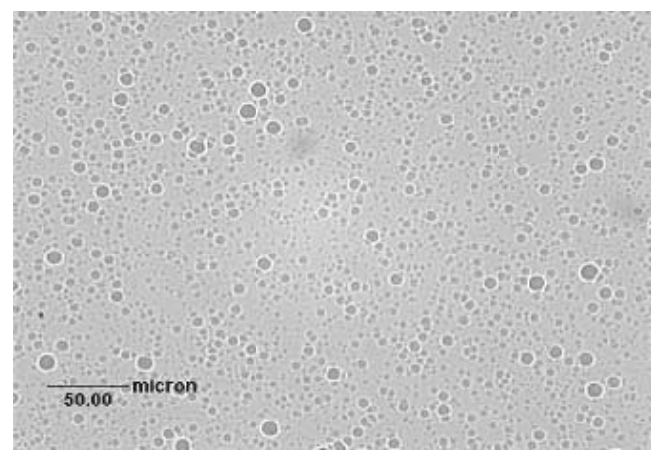

Figure 2: Microscopic image of etch pits made by ions on CR-39.

$\times 10^{18} \mathrm{~W} / \mathrm{cm}^{2}$. The fast ion emission was recorded by the CR-39 solid state nuclear track detector. The ions with the energies of more than $100 \mathrm{keV}$ per nucleon can be recorded as tracks on a surface of the detector, after being etched in sodium hydroxide solution. A Thomson parabola was used in order to separate ion energy and charge. Generated ions coming into the Thomson parabola would feel an electric force and a Lorentz force due to the electric field and the magnetic field which were applied parallel to each other. Ions' trajectories would curve and separate due to differences in energy and charges. Figure 2 shows a typical image of CR-39 irradiated by energetic ions generated from a $\mathrm{Cu}$ target with the laser power of $\sim 7$ TW. Sizes of etch-pits were $2 \mu \mathrm{m}$ to $8 \mu \mathrm{m}$. We compared them with etch-pits made by accelerated ions with specific energies from a Tandem Van de Graaff accelerator. Inferred energies of laser-generated fast ions were $0.2 \mathrm{MeV}$ to $3 \mathrm{MeV}$. The number of generated ions was $6 \times 10^{4} \mathrm{particle} / \mathrm{sr} / \mathrm{shot}$. Figure 3 shows ion spots put on a CR-39 located behind the Thomson parabola. A pin hole with the diameter of 1 


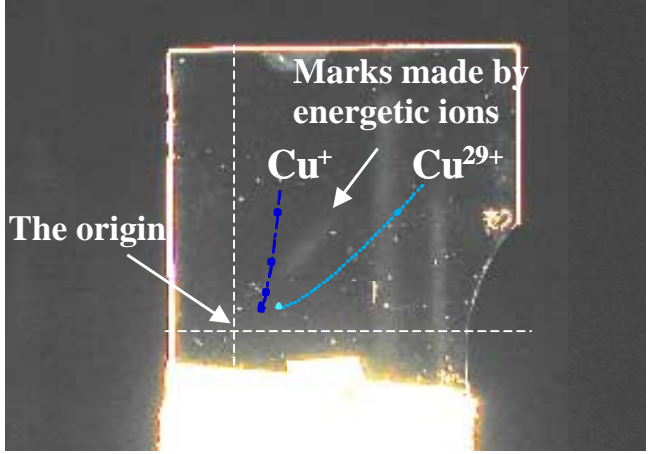

Figure 3: Measurement with a Thomson parabola.

$\mathrm{mm}$ was located in front of the Thomson parabola to have a better resolution. 2.5 TW laser was used for ion generation. We can see marks printed by energetic ions within the region of $\mathrm{Cu}^{12+}$ to $\mathrm{Cu}^{17+}$ and $50 \mathrm{keV}$ to $220 \mathrm{keV}$.

\section{X-RAY GENERATION AND TIME-RESOLVED X-RAY DIFFRACTION}

The outline of the time-resolved X-ray diffraction system at NERL is shown in Fig. 4. The laser pulse coming into the vacuum chamber is divided to the main pulse and the pump pulse in a ratio of nine to one by the beam splitter. The former is focused onto the copper target with the off-axis parabolic mirror (focal length $=162 \mathrm{~mm}$ ) to generate the laser produced plasma X-rays that are used for X-ray diffraction from the single crystal as the probepulse. Focused by the spherical lens, the latter induces transient phenomena inside the crystal after passing through the delay-path with which the time interval between the pumpand probe- pulses can be controlled. For easy alignment, large wafers or disks of single crystals are employed as the samples for X-ray diffraction and mounted on the automatic positioner. The two-dimensional diffraction images are taken by X-ray imaging plates with the spatial resolution of $50 \mu \mathrm{m}$. The samples are surrounded by lead plates for shielding of the background X-rays scattered from the circumference. The X-rays may reach the sample surface only through the 3-mm-wide slit. Polyvinylidene chloride film is put in front of the parabolic mirror as a protection against ablated debris from the copper target. Diffracted Xrays pass through the beryllium window and are measured by the imaging plates outside of the chamber. Another 1$\mathrm{cm}$-wide slit is put at the exit of the beryllium window to reduce the background $\mathrm{X}$-rays.

Figure 5 shows diffraction images for GaAs wafer of (111) orientation taken with an imaging plate with delay times of $0 \mathrm{ps}$ and $150 \mathrm{ps}$ with the laser fluence of 1.3 $\mathrm{mJ} / \mathrm{mm}^{2}$. Two lines on images in Fig. 5 are composed of the characteristic X-rays of copper, $\mathrm{K} \alpha 1$ and $\mathrm{K} \alpha 2$. The accumulation time of these images was about $30 \mathrm{sec}$ at 10 $\mathrm{Hz}$ repetition. The laser energy of $350 \mathrm{~mJ}$ per pulse was

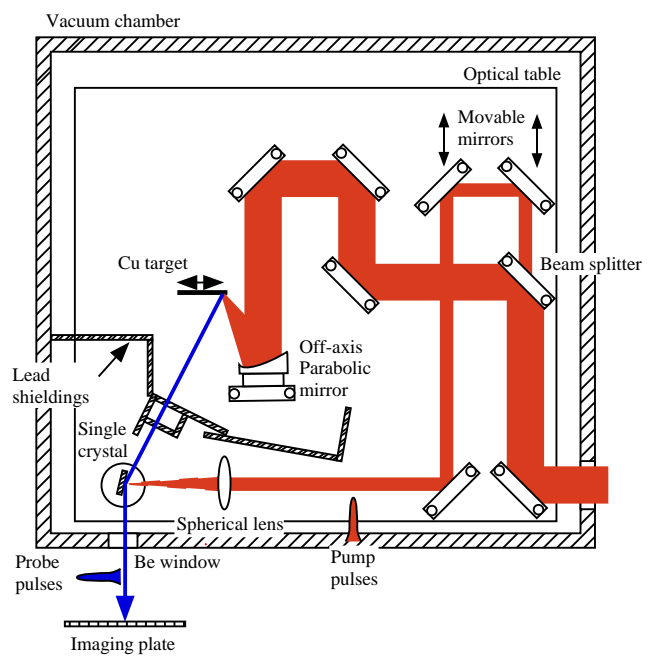

Figure 4: Experimental setup for time-resolved X-ray diffraction.

devoted to the X-ray generation. At 0 ps delay, we see no deviation in the image and there are only two lines in the same way as diffraction from an unperturbed crystal. At $150 \mathrm{ps}$ delay, there appears another line on the left side of the original lines within the laser-irradiated region of the image.

Figure 6 shows horizontal profiles of the diffraction intensities at $0 \mathrm{ps}$ and at $150 \mathrm{ps}$. In addition to two peaks that consist of $\mathrm{CuK} \alpha 1$ and $\mathrm{CuK} \alpha 2$, we can see the subpeak that results from the lattice deformation induced by the laser pulse irradiation. This subpeak appears on the side of smaller angles compared with the original Bragg angle, which implies lattice expansion inside the crystal.

The laser coming into the crystal will excite electrons in the valence band to the conduction band and thus undertake absorption by making electron-hole pairs. Therefore energy deposit will be distributed according as this absorption length. Then energy transfer from carriers to lattice will occur by scattering. This brings rapid temperature increase in the crystal, resulting in thermal stress into the lattice. This thermal stress triggers a strain wave propagating

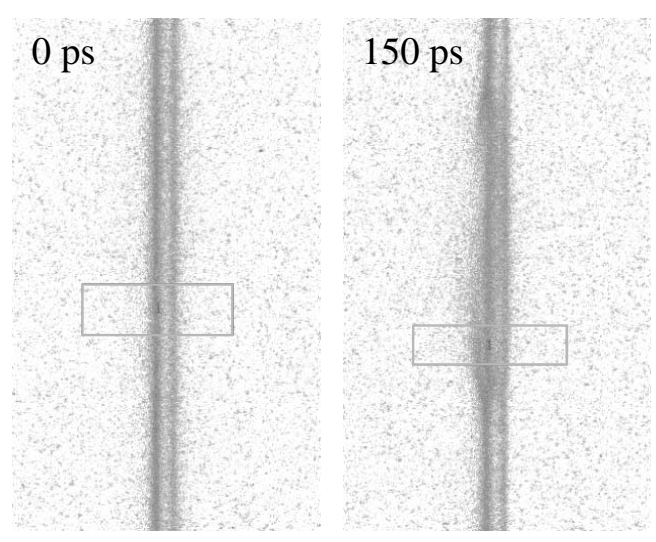

Figure 5: Diffraction images detected with imaging plates. 

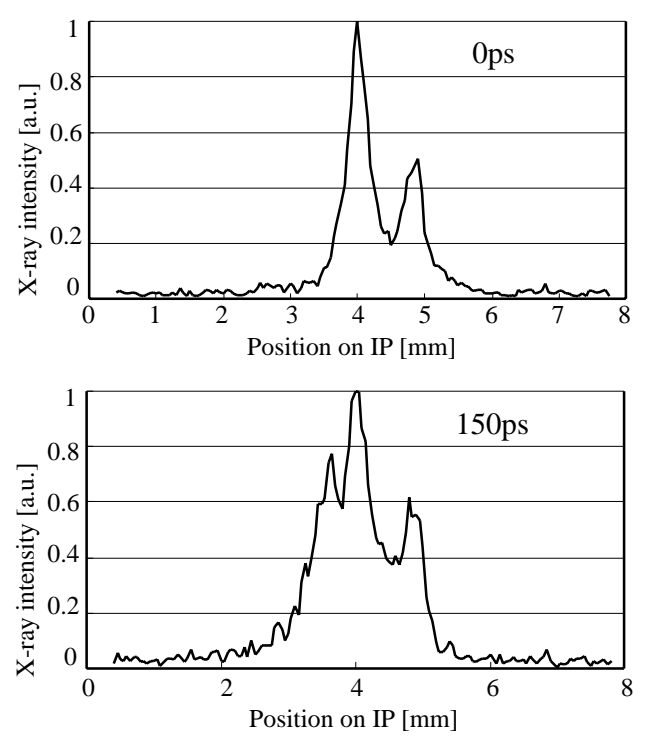

Figure 6: X-ray diffraction profiles.

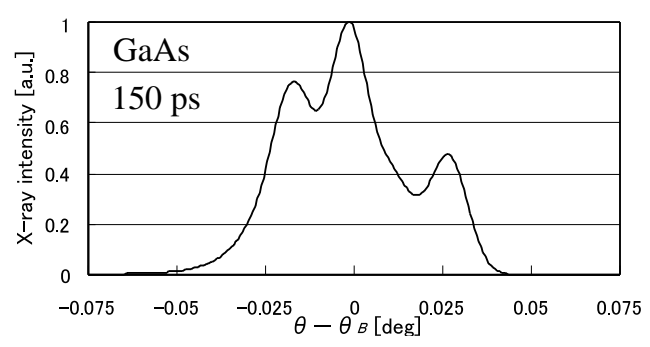

Figure 7: Simulated result of X-ray diffraction profile.

into the crystal with the speed of sound. Thus, the strain distribution that results from the above process is given by the following equation [2].

$$
\begin{aligned}
\eta_{33}(z, t)=\Delta T_{s} & \frac{1+\nu}{1-\nu}\left[e^{-z / \zeta}\left(1-\frac{1}{2} e^{v t / \zeta}\right)\right. \\
& \left.-\frac{1}{2} e^{-|z-v t| / \zeta} \operatorname{sgn}(z-v t)\right],
\end{aligned}
$$

where $\eta_{33}$ is the $z z$ component of the strain tensor, $\Delta T_{s}$ is the temperature increase at the surface, $\nu$ is the Poisson ratio, $z$ is the distance from the surface inward to the crystal, $\zeta$ is the absorption length for the laser pulse, $v$ is the speed of sound in the crystal, $t$ is the passed time after the laser irradiation. Figure 7 is the calculated result for the crystal with the strain expressed by Eq. 1, based on the kinematic diffraction theory. Here also we can see the subpeak that behaves in the same manner as the experimental one, as well as peaks of characteristic X-rays.

\section{CONCLUSION}

We successfully generated energetic ions and X-rays with an ultraintense laser interacting with a solid target. Fast ions of copper that have the energy from $0.2 \mathrm{MeV}$ to $3 \mathrm{MeV}$ were detected with CR-39. Using a Thomson parabola, it was confirmed that the copper ions were ionized with $12+$ to $17+$. Picosecond temporal evolution of Xray diffraction from GaAs(111) single crystals was demonstrated by the time-resolved X-ray diffraction using ultrashort X-rays from laser produced plasmas. Those energetic particles may provide a useful tool for ultrafast material analyses.

\section{REFERENCES}

[1] A. Zhidkov, A. Sasaki, T. Tajima, "Emission of MeV multiple-charged ions from metallic foils irradiated with an ultrashort laser pulse", Phys. Rev. E 61 (2000) R2224-R2227.

[2] C. Thomsen, H. T. Grahn, H. J. Maris, J. Tauc, "Surface generation and detection of phonons by picosecond light pulses", Phys. Rev. B 34 (1986) 4129-4138. 